\title{
AN IMPROVED POWER SUPPLY SYSTEM FOR NEUTRAL SECTIONS IN RAILWAY ELECTRIC POWER SYSTEMS
}

\author{
IN KWON PARK ${ }^{1}$, BYOUNG-GON LEE ${ }^{2}$ JU-RAK KIM ${ }^{3} \&$ JANG-MU LEE ${ }^{3}$ \\ ${ }^{1}$ RTDS Technologies Inc. Canada \\ ${ }^{2}$ KORAIL, Korea \\ ${ }^{3}$ Korea Railroad Research Institute, Korea
}

\begin{abstract}
In order to ensure the necessary level of electrical isolation between neighbouring substations, it is essential that the neutral sections should be installed. The neutral section provides the necessary electrical isolation between the substations. An added benefit of such configuration is that it can offer the flexibility of the changing the source of power to certain feeder segments. If necessary, individual feeder segment can be cut off from its main power while the continuation of the feeder service is guaranteed by a nearby substation. However, the existence of the neutral section in the feeder line means the loss of traction power when an electric locomotive passes through the section. Because of no traction power, the speed of the train cannot be regulated. Usually, the speed slows down during the passage of the section. Such loss of the speed might introduce a significant amount of delay in the total travel time of the train. Moreover, if the speed of the locomotive happens to be almost standstill when it enters the section, then there is a good chance to see that it would stop in the middle of the section. Then, it is unable to escape the section unless a helping locomotive is to be dispatched there to draw the train out of the section. Therefore, there has been much attention and research effort into the issue of ensuring proper power supply to the section. In this paper, an improved approach to the power supply system to the neutral section is proposed. In this approach, a modern power electronics topology of MMC (Modular Multi-level Converter) is introduced as part of the main power supply system. The effectiveness of the proposed system was verified through realistic real-time simulation results.

Keywords: railway electric power system, neutral section, modular multi-level converter, phase-shift control.
\end{abstract}

\section{INTRODUCTION}

Once a particular electrical railway line becomes longer than a certain extent, a single substation can no longer supply the necessary electric power for the entire line, from the beginning to the end. If a single substation is put in place at the one end of such line and supplies the necessary power for the entire line, then the other end of the line would suffer the excessive voltage drop due to the long feeder line length and the resulting high value of line impedance. Thus, such configuration becomes unable to provide the necessary power to the railway tractors or EMUs operating on the line. Therefore, placing multiple the supply stations along with the line with a regular interval is customary in the electrified railway system configuration. Usually, each of those supply stations is in charge of a line length between 15 and $30 \mathrm{~km}$. With such configuration along with the line, it becomes apparent that each of those stations needs to be isolated from one another. In the electric power system, there is good chance to see that the supply lines to those stations are from different sources, causing different voltage magnitude and phase angle values. If two supply stations where different voltage magnitude and phase angle values exhibit get connected together, practically what happens is an electric short circuit fault, which would result in severe damage to the electrical equipment such as transformers on both stations. Furthermore, such fault would cause a disruption in the power supply to the railway line, which would disturb the operation schedule of the railway line. Hence, it is imperative to ensure that each of those supply stations in the railway line is isolated from the neighbouring stations. One easy and 
practical solution for the issue of isolation is placing a neutral section between two-line segments. The neutral section is not connected to any of those two stations nearby. Thus, the section itself does not have any voltage. Therefore, two adjacent line sections at both ends of the neutral section can be supplied by substations with different voltage magnitude and phase values. Fig. 1 shows the concept of the neutral section.

One obvious consequence of having the neutral sections in the line is that no power can be delivered to a traction system during the train is going through the section. Therefore, the train loses the ability to regulate the speed except braking using its own system independent from the feeder line, such as a mechanical brake. Furthermore, the HVAC system and the control system should be put into a limited power source either from an onboard battery or the regeneration by utilizing the existing kinetic energy of the train. Inevitably the train loses the speed while it goes through the section. One literature published in China [1] stipulated that more than 30 minutes of travel time can be saved if all of the neutral sections in the highspeed railway line between Beijing and Shanghai (total length of $1318 \mathrm{~km}$ ) can be eliminated. More severe consequence one can imagine is the possibility of a train's being trapped in the section. If a train enters the section with very slow speed, then the inertia of the train might not be enough to push the train throughout the section and make it escape at the other end. Unless another helping traction car can be dispatched and pull or push the trapped train to outside, the train cannot move. Once the train becomes trapped, it blocks the entire line, preventing the regular and timely operation of other trains on the same line.

In order to overcome such difficulty associated with the neutral section, various approaches have been attempted. The core idea of such attempts is how to supply power to the neutral section while a train is going through it. The following figure, Fig. 2, presents one such attempt. In this attempt, the neutral section in the middle is connected to both sides of the section through high-speed circuit breakers (S1 and S2 in the figure). Those circuit breakers are usually vacuum circuit breakers. Before the train comes close to the section, the breaker S1 is ON, and the breaker S2 is off. Thus the section is at the same voltage potential as the line at the left to the section. When the train passed through the point $\mathrm{B}$, the breaker $\mathrm{S} 1$ turns off, and the breaker S2 turns on. Thus, the train begins to receive the power from the line right to the section. In this way, the amount of time the train experiences no power can be minimized.

However, the drawback of this approach is also evident. When the switches change the ON/OFF state, the voltage applied to the neutral section suddenly changes. Such sudden

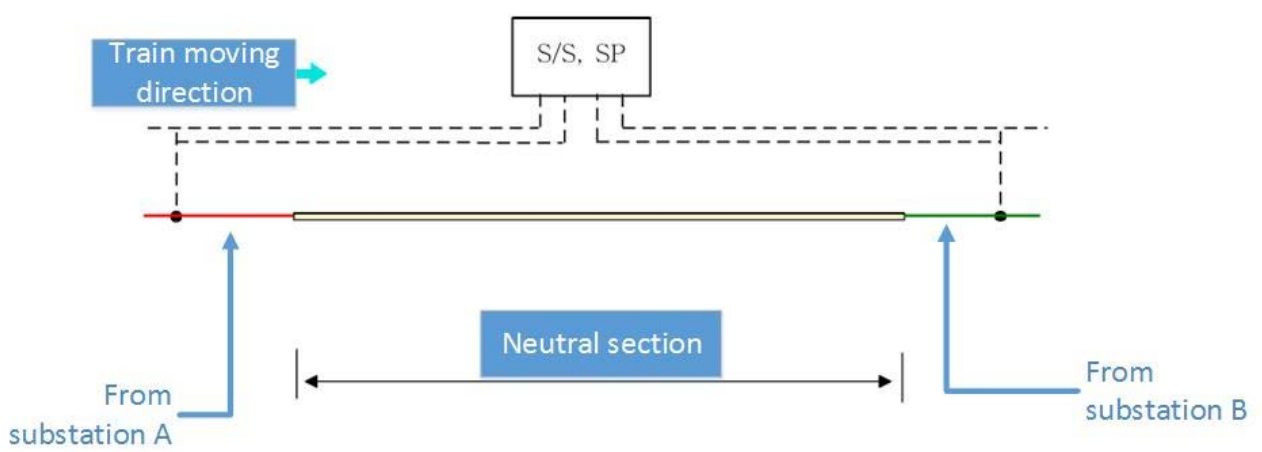

Figure 1: A neutral section. 


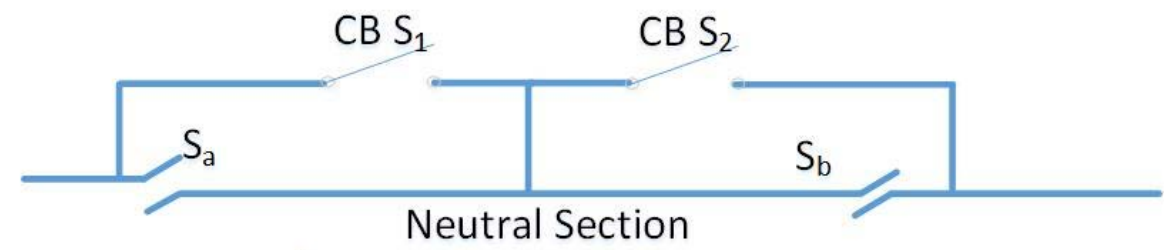

Train moving direction

Figure 2: A way to supply power to a neutral section [2].

change can cause an adverse impact on the onboard electrical system. In the worst scenario, the new voltage magnitude and the phase values coming from the pantograph when the switching happens can induce a fault like a circumstance across the main transformer on the train, causing the tripping of the main onboard circuit breaker, cutting off the entire power supply to the train.

A new way of supplying power through the modular multilevel converter (MMC) is proposed in this paper. The proposed power supply system can provide the voltage magnitude and phase angle in an arbitrary manner, independent of those values at the nearby lines. Therefore, the system can provide the power to the neutral section with the gradually changing magnitude and the phase value while a train is going through the section. In other words, the power supply system voltage magnitude and phase value can start from the same value of the line which is left to the section and gradually change the value up to the new value which is the same as the one from the line at the right side, assuming the direction of movement is in Fig. 2. Such gradual transition during the train's traverse through the section can suppress the impact of the change to the minimum level, reducing the possibility of damaging the onboard electrical system. The effectiveness of the proposed system is verified by the results from real-time digital simulations.

The rest of this paper is laid out in this way: In the following chapter, the concept of the proposed neutral section power supply system is explained. The simulation results of the proposed system follow the explanation. The concluding remark section at the end completes this paper.

\section{PROPOSED NEUTRAL SECTION POWER SUPPLY SYSTEM BY USING A MODULAR MULTI LEVEL CONVERTER (MMC)}

With the given advantage of the MMC system, composing a new neutral section power supply system is proposed. The primary goal of the system is to offer the capability of a smooth transition of the supply voltage between the adjacent substations. In the existing systems, it was noticed that such smooth transition was not possible. Therefore, the traction system in a train inevitably suffers the substantial amount of supply voltage changes, in terms of the magnitude and the phase angle. As a result, frequently the main circuit breaker on the train tripped and cut the power supply to the entire traction system, making the reduction of the train speed due to the loss of power much worse. Furthermore, when the voltage magnitude and phase angle change happens on that scale, it is likely to see that particular 
devices in the onboard power conversion system including the main transformer would receive the damage. Subsequently, the performance would become degraded with multiple shocks they would suffer from passing the neutral section many times during the operation. Eventually, aggravation of such damage would induce premature failure of the devices, raising up the cost of operation unnecessarily and bringing unexpected stoppage of the operation. In short, the proposed system was devised with the two goals in mind:

1. Continuous and uninterrupted supply of the power to the train when it passes through the neutral section

2. The smooth transition of the voltage at the pantograph, in terms of the magnitude and phase angle

The overview of proposed power supply system is shown in Fig. 3.

\subsection{Power stack}

The power conversion system is composed of two separate power electronics bridge systems. The first one is a 2-level bridge with a high pass filter. The second one is an MMC based multilevel bridge. Those two bridges are interconnected together through a shared DC stage. The 2-level bridge at the left side of the system is connected to the substation A through a transformer. In order to suppress the total amount of generated harmonics from the 2-level bridge, going into the AC system, a high pass filter was installed at the AC stage of the bridge. The equations, from eqns (1)-(4) below, show how the circuit parameters of the high pass filter were calculated.

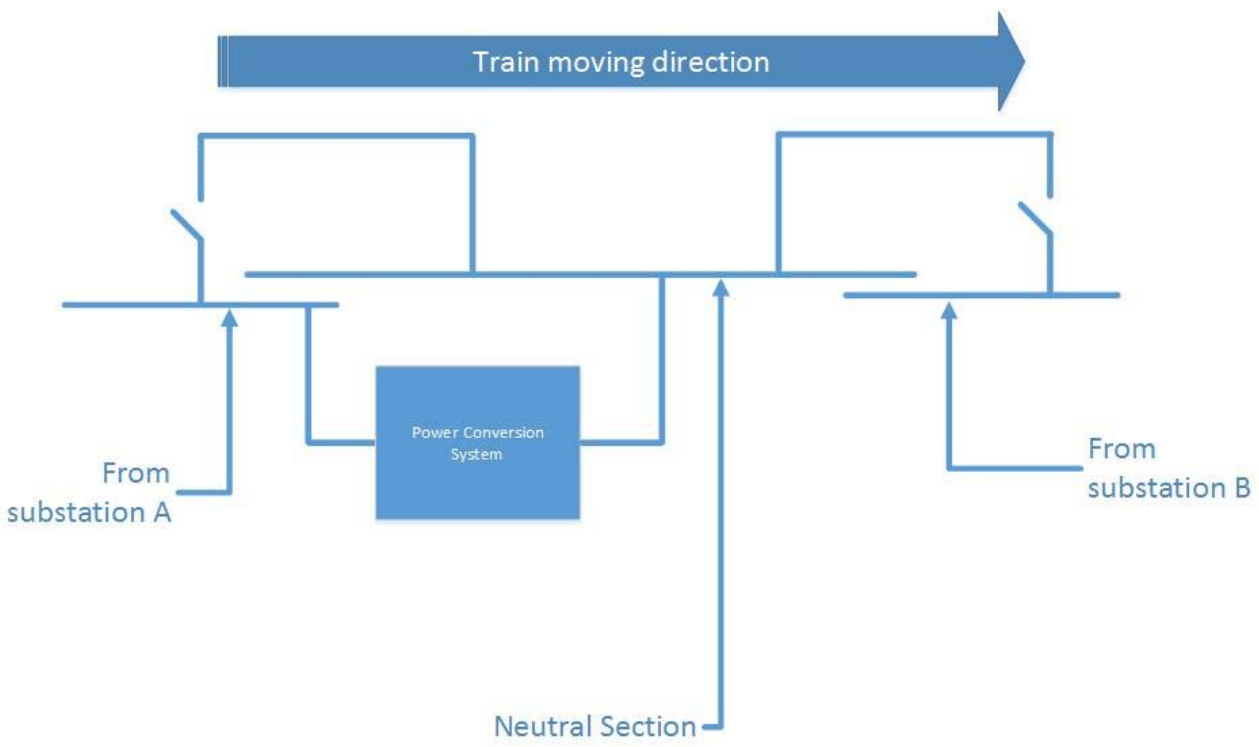

Figure 3: Proposed neutral section power supply system - overview. 


$$
X_{C}=\frac{(\text { V-line to neutral } R M S)^{2}}{\text { Filter MVAR rating }}
$$

$$
\text { Capacitance }=\frac{1.0}{2.0 \cdot \pi \cdot(\text { Base Frequency in } \mathrm{Hz}) \cdot X_{C}}
$$

$$
\text { Inductance }=\frac{1.0}{(2.0 \cdot \pi \cdot \text { Cutoff Frequency in } \mathrm{Hz})^{2} \cdot \text { Capacitance }},
$$

$$
\text { Resistance }=(2.0 \cdot \pi \cdot \text { Cutoff Frequency in } \mathrm{Hz}) \cdot \text { Inductance. }
$$

In order to get the necessary filter circuit parameters using eqns (1)-(4), the high pass filter cut-off frequency was set to the same frequency as the switching frequency of the 2level bridge, which was $2 \mathrm{kHz}$. Then, the rated voltage was set to the transformer secondary voltage, and the rating was set to around one-third of the transformer rating (10 MVA).

The 2-level bridge regulates the DC capacitor voltage at the DC stage in the middle. Then, the $\mathrm{DC}$ voltage becomes the positive $\mathrm{DC}$ rail and the negative $\mathrm{DC}$ rail voltage to the $\mathrm{MMC}$ bridge at the right side. If the train entering the neutral section is in the traction mode, then the power should be supplied from the power supply system to the traction system in the section. In such case, the power flows from the station A, then it goes through the 2-level bridge and comes to the MMC bridge. Then, the MMC bridge delivers the necessary power to the traction system connected to the section line through its pantograph. The DC stage voltage takes the vital role of detecting the power flow. If the DC voltage goes lower, which would happen when the power from the 2-level bridge is less than the power delivered to the train through the MMC bridge, then it should be restored by proper control action of the 2level bridge. The details of the necessary control is explained further in the next section.

On the other hand, the flow turns to the opposite direction when the train entering the section tries to slow down by regenerative braking. In such case, the kinetic energy the train carries by its speed and weight transforms into the electric power through the regenerative braking action of the traction motor and its drive system. Then, the power coming from the drive system flows into the line of a section through the train's pantograph. Then, the power flows into the MMC bridge, continuing its flow through the 2-level bridge at the left side. The flow ends up at the line connected to the station A. Again, the DC voltage in the middle can signify the power balance between the MMC bridge and the 2-level bridge. If the power coming from the MMC bridge is larger than the power taken by the 2-level bridge, the voltage would rise. With the proper control, the voltage can be regulated with a reference value, indicating that the power flowing from the MMC bridge is balanced with the power flowing out through the 2-level bridge.

Of course, one can ask that both bridges can be configured by the same type of topology, either two 2-level bridges or two MMC bridges. However, such configuration would be less preferable to the one proposed in this paper. The following points would justify the proposed topology. First, the 2-level bridge output waveform would produce a rich set of harmonics. Thus, suppressing the harmonics by utilizing filter becomes imperative in the case of the 2level bridge. Then, such utilization of the filter slows the control and synthesis of the output voltage waveform. However, the 2-level bridge would offer the economics in the construction and maintenance. The large number of components which comprise the MMC bridge becomes a liability in that regard. Thus, such topology, namely a 2-level bridge, would be more suitable for a system which is to be interfaced to a steady AC system. In the proposed system, such stable AC system can be found at the power source side, the station A line in 
Fig. 3. Meanwhile, while a train is passing through the section, maintaining smooth transition in terms of the voltage magnitude and the phase angle becomes more important. Otherwise, the proposed neutral section power supply system loses its ground. Then, in order to make sure the intended purpose is to be attained, the high bandwidth in terms of controlling the output voltage magnitude and phase angle becomes imperative. In other words, the bridge facing up to the neutral section needs to fulfill the necessary speed of control, which would be more difficult with 2-level bridge configuration and its companion filter. The need for the control speed can be demonstrated with a simple example. In certain electric railway systems, the length of the section can be as short as $47 \mathrm{~m}$ [3]. Assuming that a high-speed train passes through such short section with its full speed $(300 \mathrm{~km} / \mathrm{h})$, the time between the moment when a pantograph enters the section and the moment when it leaves is around $0.56 \mathrm{sec}$. Therefore, the neutral section power supply system needs to fill up the difference in terms of the voltage magnitude and phase angle within such short period. Hence, such requirement is asking for the high-speed power electronics-based bridge like the one in the proposed system in this paper.

\subsection{Control}

As briefly touched previously, the control of the power stack plays an important role in achieving the purpose of the system. The control of the entire system can be split into two, the first for the 2-level bridge and the second part for the control of the MMC bridge. The overall idea of controlling the single phase 2-level bridge came from [4]. One fundamental difference between a single-phase system and a three-phase system is that there is no inherent 2 degree of freedom in the single-phase system. Therefore, the quadrature signal, which would be perpendicular to a certain input value, needs to be generated in an artificial way. In the reference [4], such quadrature signal generation was achieved by using all-pass filters. However, the all-pass filter can impede the speed of the control. Therefore, a different approach, a SOGI based quadrature signal generation method [5] was used in the control implementation of the proposed system. The following figure, Fig. 4, shows such quadrature signal generation from the input current signal.

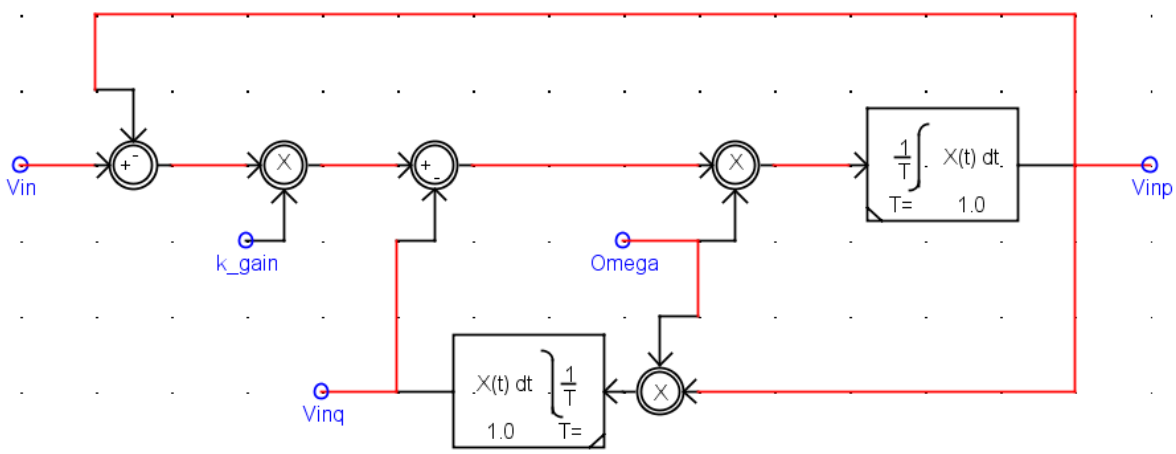

Figure 4: A SOGI-QSG (Quadrature Signal Generator). 
Another significant role of the control is detecting the correct phase of the AC voltage. Such information, the phase angle of the AC voltage waveform becomes the reference for the rest of the control systems. In the control implementation of the proposed system, a wellknown single-phase PLL implementation (ePLL) [6] was employed to ensure the expected level of performance from the system. With the information from both SOGI-QSG and the ePLL, a traditional 2-axis control (D-axis and Q-axis) was implemented to control the real power flow and the reactive power flow through the 2-level bridge. The control objective of the real power flow was given as the regulation of the DC stage voltage. In other words, the real power control of the 2-level bridge attempted to regulate the voltage to a given reference value. The proper control of the real power flow control regardless of the train's mode operation (either traction mode or regenerative braking mode) can be achieved in this way. The details of such control were already introduced in the previous section regarding the power stack. Meanwhile, the reactive power control objective was left for the user's own control, allowing a user to command a certain reactive power output from the 2-level bridge.

In comparison with the aforementioned 2-level bridge control, the MMC bridge control is simpler. Again, the purpose of the proposed neutral section power supply system is to ensure the smooth transition of the voltage magnitude and phase angle between the substation A and substation B, which are isolated by the neutral section. Therefore, two types of the information are necessary for the control of the MMC bridge, the phase information on both sides of the section and the voltage magnitude information. The phase information can be obtained in the same way as described in the previous 2-level bridge control, presented in. Then, the voltage magnitude values from both sides are obtained from the control loop presented in Fig. 5.

In this implementation, the system frequency was assumed to be fixed at $50 \mathrm{~Hz}$. The integrator reset signal, a signal named "sinethetax" is from the PLL which takes the same input as the RMS calculation routine in Fig. 5. The following figure, Fig. 6, shows the core control of the MMC bridge.

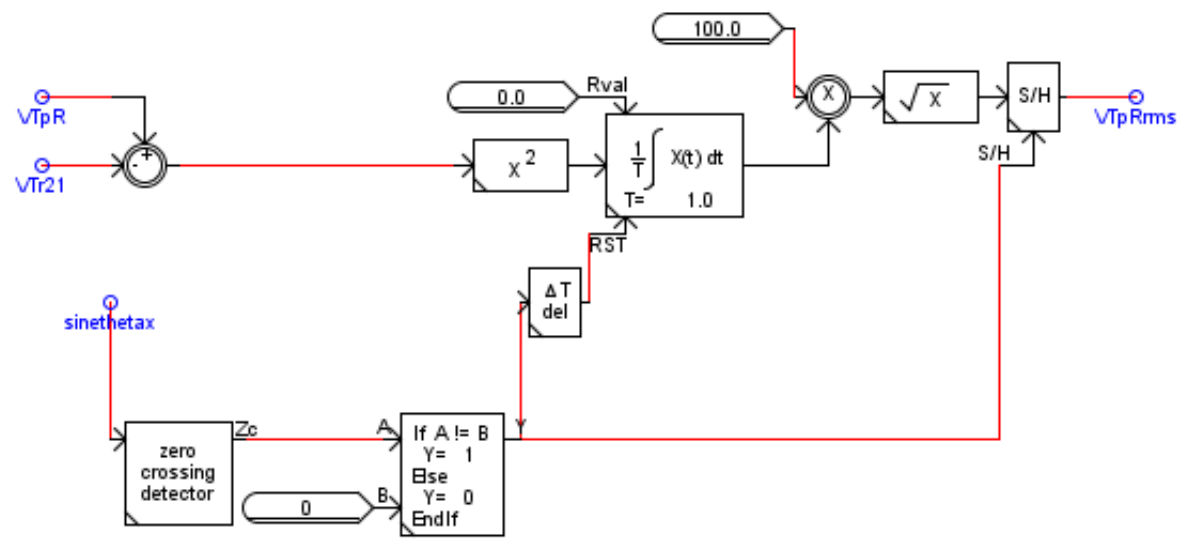

Figure 5: A single-phase RMS meter. 


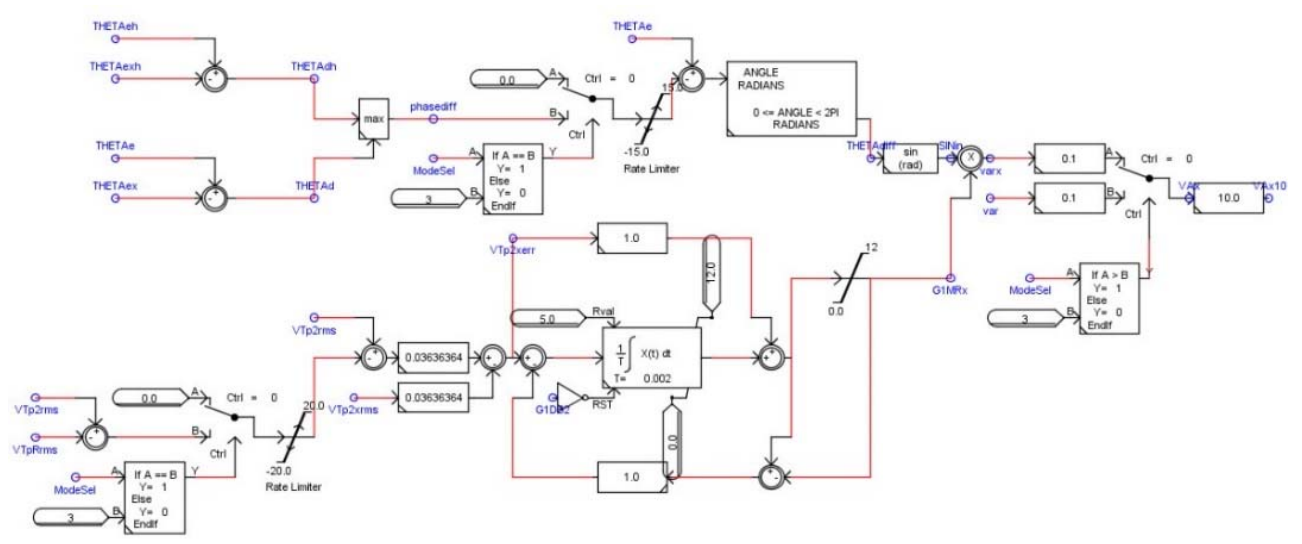

Figure 6: MMC bridge control.

\section{PROOF OF THE EFFECTIVENESS BY SIMULATION RESULTS}

In order to verify the effectiveness of the proposed system, a real-time simulation case was constructed, and the simulation results were checked. The simulation platform is RTDS ${ }^{\mathrm{TM}}$, where the simulation case can run in real time. Thus, one does not need to wait too long before the simulation results become available. This fast (real time) execution of the simulation offers a substantial advantage over the off-line simulation tools, where sometimes an excessive amount of time would be consumed to run a realistic simulation case. The time requirement of the off-line type simulation tools gets aggravated when complex power electronics system comes in as part of the simulation case. The proposed neutral section power supply system contains a 2-level bridge as well as an MMC bridge. The behavior of those bridges has to be modeled and simulated in the necessary level of details. Such requirement brings the heavy load of calculation, which would entail excessive simulation time in an off-line simulation tool. However, because of the real-time simulation capability available, the simulation time can be shortened. Therefore, many iterations of the development steps can be accommodated by utilizing the real-time simulation platform in the necessary simulation work, as one used in this verification.

The following figure, Fig. 7, shows the overview of the constructed simulation case. Both substations in the simulation case were modeled as a single-phase voltage source with impedance. Then, the train was modeled as a resistive load in the middle. This modeling approach is for the occasion when the train enters the section in a traction mode. Thus, a certain resistance which represents the power consumed by the train was used as the necessary representation of the train. The traction load was assumed as $2 \mathrm{MW}$ in this simulation case. Then three breakers, labeled as "BRK1A", "BRK1B" and "BRK1C", controls the sequence of the event which would simulate the train's movement through the section. When the breaker "BRK1A" is ON and the rest are OFF, the load is connected to the substation A. This step represents the moment when the train is approaching the section, but not reach the section yet. Then, when the breaker 'BRK1B' is ON and the rest are OFF, the load is connected to the neutral section, representing the train's passing through the section. Then when the breaker "BRK1C" is ON and the rest are OFF, the load becomes connected to the substation B. 


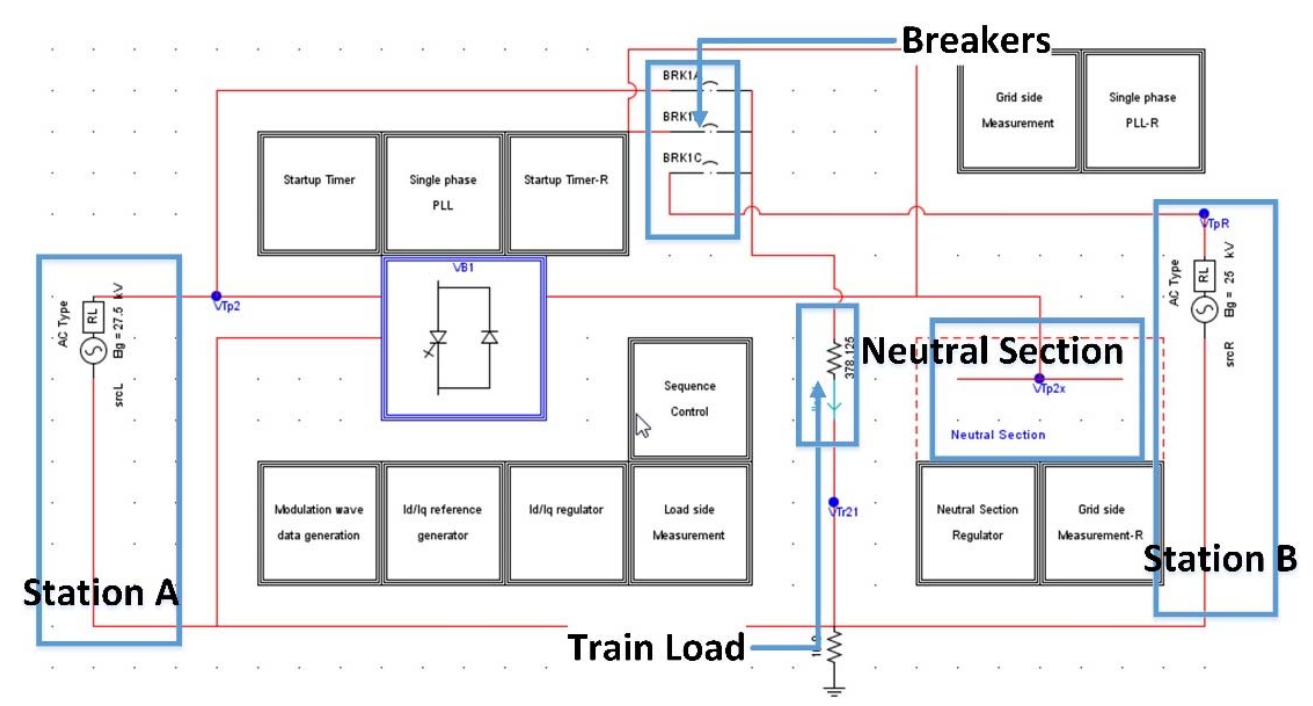

Figure 7: An RTDS neutral section power supply system simulation case.

The following figure, Fig. 8, presents the simulation results. The pulses, labelled as 1, 2, 3 and 4 , point the start of a step in a series of events. The pulse 1 signifies the moment when the train is approaching the section; A signal is sent to the neutral section power supply system to make it ready. In other words, the signal initiates the arming state of the system, making the proposed system to start to produce the voltage output in sync with the substation A voltage, where the train is taking power from. Then, the pulse 2 represents the moment when the train enters the section. From this moment until the train leaves the section, the proposed power supply system takes charge of supplying power to the train. Then the pulse 3 notes the moment when the proposed power supply system begins to change the output voltage, from the substation A reference to the substation B reference. In the simulation case, the substation A voltage source is set to the voltage magnitude of $27.5 \mathrm{kV}$ (RMS) and the phase angle of 0 degree, while the substation B voltage source is set to the magnitude of 25 $\mathrm{kV}$ (RMS) and the phase angle of -90 degrees. Such difference is readily noticeable in the first graph of Fig. 8, where the waveform labeled as "VTp2' represents the substation A voltage, while the waveform labeled as "VTpR' represents the substation B voltage. Then, finally, the pulse "4' signifies the moment when the train leaves the section and enters the line segment supplied by the substation B. One obvious simulation result is the gradual decrease of the DC voltage during the train moves in the section. The waveform in the middle of shows such result. Because the train takes the traction power through the power supply system, the power flows from the 2-level bridge to the MMC bridge, taking out a certain amount of energy from the energy stored in the DC stage in the middle of the power supply system. Consequently, the voltage would drop as more energy is drawn from the DC stage. The transition completes less than 6 cycles, $120 \mathrm{~ms}$ in $50 \mathrm{~Hz}$ system. Such period can be translated to the distance of $10 \mathrm{~m}$ when a train speed is at $300 \mathrm{~km} / \mathrm{h}$. Thus, this simulation result shows that the proposed power supply system can still satisfy its given goals when the length of the section becomes extremely short. 


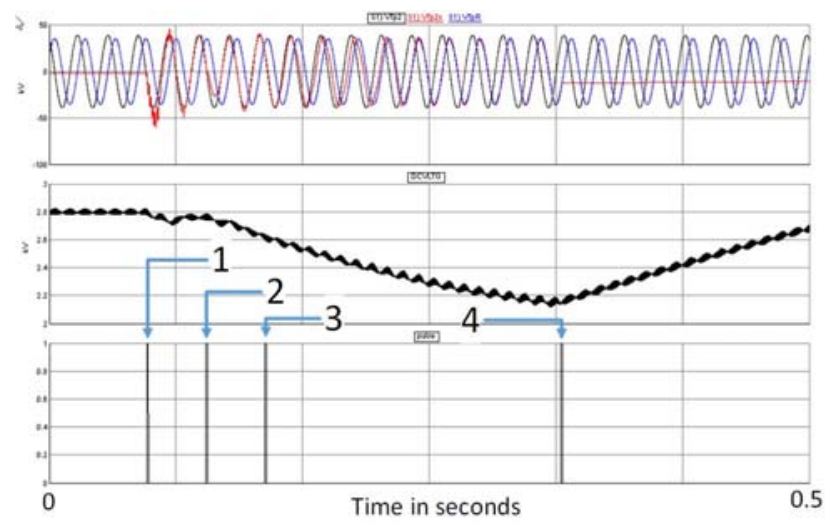

Figure 8: Simulation results: traction mode.

The next figure, Fig. 9, shows another simulation results when the operating mode of the train was modeled as regenerative braking mode. Again, the obvious simulation result indicating the train in the section is in the operating mode, a regenerative braking mode, is the gradual rise of the DC voltage. The direction of the power flow between the train and the power supply system reverses when the train is in the operation mode. The power is generated by the train, then it comes into the MMC bridge, and it flows into the 2-level bridge. Thus, the DC stage in the middle stores the extra energy coming from the regenerative braking. Such extra energy at the DC stage raises the voltage, which is clearly shown in the middle graph in Fig. 9. Probably the magnitude of the DC voltage change can be adjusted by changing the circuit parameters and the control parameters, but no such effort was attempted in this paper. Then, as noted in the simulation results previously explained, the proposed power supply system successfully changes the voltage output from the one reference (from substation A) to the other reference (from substation B) in a smooth way. Therefore, the simulation results introduced here as well as the one previously presented proves that the proposed neutral section power supply system can fulfil its intended purpose.

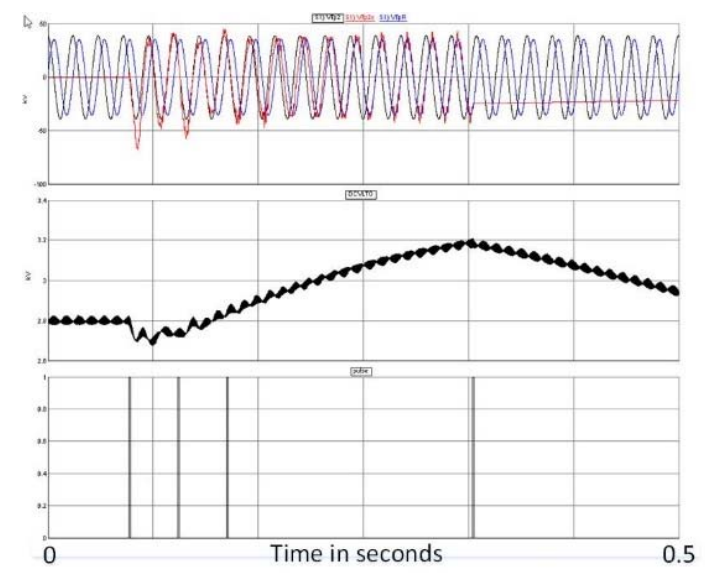

Figure 9: Simulation results: regenerative braking mode. 


\section{CONCLUDING REMARKS}

An improved neutral power supply system in an electric railway system is proposed in this paper. The two objectives of the proposed power supply system are to maintain the power supply to the electric train when it passes through the neutral section and the transition from the one side to the other side smoother. Such objectives would eliminate the speed loss of the train due to the lack of power it suffers whenever it passes through the sections and reduce the shock the onboard system would suffer when no mitigating effort is made. The power stack of the proposed system is composed by the combination of a 2-level bridge and an MMC bridge. The MMC bridge at the neutral section side enables the smooth and speedy transition of the voltage output from the one side of the section to the other. The effectiveness of the proposed system was verified by real-time simulation results. It is expected that further research would bring the proposed system closer to the practical application in a real electric railway system.

\section{REFERENCES}

[1] Tian, X., Jiang, Q. \& Wei Y.-D., Research on novel uninterruptible phase-separation passing scheme in electrified railways. Power System Protection and Control, 40(21), 2012. (in Chinese.)

[2] Sun W.-H., Dan S.-N. \& Zheng G.-F., Comparison of domestic and foreign manufactured automatic phase-separation passing schemes. Electrified Railway, 2002. (in Chinese.)

[3] KORAIL, Neutral section types and installations in KORAIL. (in Korean.)

[4] Rae-Young, K., See-Young, C. \& In-Young, S., Instantaneous control of average power for grid tie inverter using single phase D-Q rotating frame with all pass filter. Industrial Electronics Society, 2004. IECON 2004. 30th Annual Conference of IEEE, 1, pp. 274279, 2004.

[5] Ciobotaru, M., Reliable Grid Condition Detection and Control of Single-Phase Distributed Power Generation Systems. Institute of Energy Technology, Aalborg University, 2009.

[6] Karimi-Ghartemani, M., Enhanced Phase-Locked Loop Structures for Power and Energy Applications, John Wiley \& Sons, Inc., 2014. 